Quim. Nova, Vol. 35, No. 5, 910-913, 2012

\title{
PARAMETRIC SENSITIVITY ANALYSIS FOR THE HELIUM DIMERS ON A MODEL POTENTIAL
}

\author{
Nelson Henrique Teixeira Lemes* \\ Instituto de Química, Universidade Federal de Alfenas, Rua Gabriel Monteiro da Silva, 700, 37130-000 Alfenas - MG, Brasil \\ Vitor D. Viterbo, Rita C. O. Sebastião e João P. Braga \\ Departamento de Química, Universidade Federal de Minas Gerais, Av. Antônio Carlos, 6627, 31270-010 Belo Horizonte - MG, Brasil
}

Recebido em 8/6/11; aceito em 10/11/11; publicado na web em 31/1/12

\begin{abstract}
Potential parameters sensitivity analysis for helium unlike molecules, $\mathrm{HeNe}, \mathrm{HeAr}, \mathrm{HeKr}$ and $\mathrm{HeXe}$ is the subject of this work. Number of bound states these rare gas dimers can support, for different angular momentum, will be presented and discussed. The variable phase method, together with the Levinson's theorem, is used to explore the quantum scattering process at very low collision energy using the Tang and Toennies potential. These diatomic dimers can support a bound state even for relative angular momentum equal to five, as in HeXe. Vibrational excited states, with zero angular momentum, are also possible for $\mathrm{HeKr}$ and HeXe. Results from sensitive analysis will give acceptable order of magnitude on potentials parameters.
\end{abstract}

Keywords: sensitivity analysis; quantum scattering; model potential.

\section{INTRODUCTION}

Theoretical and experimental informations are an important guide to infer about the quality of potentials, which should reproduce, within experimental error, a broad range of experimental data. Potentials can be obtained by a direct process, ${ }^{1}$ adjusting parameters to fit experimental data or in an inverse way, ${ }^{2}$ treating the data in a first place to retrieve the required potential. Fitting potential parameter to experimental data is not considered an inverse problem, but rather a direct problem.

Scattering matrix, $S$, calculation for a given potential provides the most sensitive test for the potential, for this will involves no average. Therefore small variations in the potential parameter can give measured effect in $S$, as will be discussed. Together with the variable phase method, application of Levinson's theorem will be explored. This important theorem relates the number of bound state a molecule can support, using scattering matrix calculations at negligible collision energy.,

Sensitivity analysis on the rare gas dimers potential parameters and the effect on bound state structure are considered in this work. How the spectrum is affected by variation in the long range dispersion coefficients and short range parameters will be discussed. This analysis will set tolerable values for the property under consideration and therefore are limits appropriate to any potential. The potential to be used in the present study is the general potential proposed by Tang and Toennies, ${ }^{5}$ although other potential are available, in special for the helium-helium interaction. ${ }^{6}$ Nevertheless, the Tang and Toennies potential is tabulated for several molecules and appropriate for inverting data in further studies on the subject.

The present study is also important as a preliminary study of rare gas clusters. ${ }^{7}$ Such clusters can be studied by using classical mechanics, in a procedure analogous to that used in atom-molecule clusters. ${ }^{8,9}$ For clusters composed of helium and neon quantum effects are important, although the classical approximation is feasible for clusters starting with argon. ${ }^{10}$ Information on the rare gas bound states structure will be provided and can be used as a motivation and as a previous analysis for further studies in the rare gas clusters quantum and classical dynamics. ${ }^{11}$

\footnotetext{
*e-mail: nelson.lemes@unifal-mg.edu.br
}

\section{THE VARIABLE PHASE METHOD}

For atomic collisions, the scattering matrix can be substituted by the phase shift, $\delta$. This phase shift carries information on how much a phase, for a wavefunction calculated for a real potential, is shifted compared with zero potential wavefunction. From this difference of phase, scattering quantities can be calculated. The main idea of the variable phase method (VPM) is to define a potential energy function, $E_{P}(R)$, which is the true one until a certain distance, $\rho$ and zero afterwards. Boundary conditions are applied at $\rho$ which is then moved forward. The phase shift, calculated for the parameter $\rho$, is constructed as this boundary is moved to large distance. Therefore, for the potential

$$
U(R)=\left\{\begin{array}{cc}
U_{\rho}(R) & 0 \leq R \leq \rho \\
0 & R>\rho
\end{array}\right.
$$

with $U(R)=\frac{2 \mu}{\hbar^{2}} E_{p}(R), \mu$ the system reduced mass, one seeks the solution in the form, $\phi_{\rho}(R)=\alpha(\rho) \sin (k R+\delta(\rho))$ with $k^{2}=\frac{2 \mu}{\hbar^{2}} E$. Amplitude and phase in this case depend on the parameter $\rho$. In the limit $\rho \rightarrow \infty$ the obtained phase will correspond to the true potential $E_{p}(R)$. Wavefunction continuity at the boundary gives,

$$
\frac{d \delta(\rho)}{d R}=-\frac{U_{\rho}(R)}{k} \sin ^{2}(k R+\delta(\rho))
$$

a first order differential equation for the phase. The initial condition, $\delta\left(R_{0}\right)=-k R_{0}$ in which $R_{0}$ is some cutoff initial radius, together with the end condition $\delta=\delta+\frac{l \pi}{2}$, taking way the possible centrifugal contribution, complete determine the phase shift and the scattering matrix. Equation 2 is the basic variable phase equation.

The full potentiality of the method has been emphasized by F. Calogero in a series of paper that derived the VPM for the general case. ${ }^{12}$ An equation for the scattering matrix, instead of the phase itself is also established. Nevertheless, the equation for the phase is more attractive since it assumes a real form and it is simple to interpret. Numerical examples are given by Calogero for exponential and 
constant potentials. ${ }^{13}$ The phase equation method was also popularized by F. Calogero, mainly because of his book, ${ }^{14}$ written in 1967.

The variable phase method has not been appropriate explored, even for the one dimension case. Number of bound states can be easily obtained in the limit of zero collision energy using the phase equation. This will require integrating a first order differential equation up to short distance. On the other hand one can integrate Schrodinger equation itself, and this will involve very large distance if the bound state has a small energy value. This is the case for the dimers studied here, as will be discussed. The method can also be formulated in several dimensions, ${ }^{15}$ although a comparison between this method and the well established close coupled equations ${ }^{16}$ has not been carried out

\section{RARE GAS DIMERS POTENTIAL}

Establishing the global potential energy function is the first and most crucial step when analyzing classical or quantum dynamics. The required potential energy function is usually approximated by a model potential with parameters obtained from available experimental data combined with quantum electronic calculation. ${ }^{17}$ Within another approach the potential can be obtained by inverting data directly. This will produce potentials for the experimental property under investigation. Using the inverse problem approach a high quality potential was obtained to reproduce second virial data ${ }^{18}$ and differential cross section. ${ }^{2}$ In the last case the potential was inverted using the first Born approximation. Nevertheless, to increase the accuracy of the inverted potential one has to use a more accurate theory, such as a full quantum calculation.

Model potentials have to be flexible to accommodate a broad range of molecules. That is the case for the potential described in ref. 17 which uses a hyperbolic tangent to damp the long range terms although this term has contribution to the short range as well. Another potential described by Slawomir and Toczylowski, ${ }^{27}$ is also used to discuss bound state energies in rare gas dimers, limited from helium to argon species. This potential is restricted to these species and cannot be used for other rare gas molecules.

A more recent and general potential with a damping function with zero contribution at short distance was developed by Tang and Toennies ${ }^{5}$ in the form,

$$
E_{p}(R)=A e^{-b R}-\sum_{n=3,4,5}\left(1-f_{2 n}(b R) e^{-b R}\right) C_{2 n} R^{-2 n}
$$

with $f_{m}(R)=\sum_{p=0}^{m} \frac{x^{p}}{p !}$ Parameters for the 21 diatomic rare gas molecules are given in literature..$^{5}$ The parameters A and b, together with dispersion coefficients are tabulated. The damping function depends only on the $b$ parameter. These five parameters, therefore, completely define the potential. Among the 21 molecules available, the present work will concentrate on the molecules, $\mathrm{HeHe}, \mathrm{HeNe}, \mathrm{HeAr}, \mathrm{HeKr}$ and $\mathrm{HeXe}$. This set of molecules will be sufficient to give confidence for further studies on the subject. Data for this set of molecule, ${ }^{5}$ are as in Table 1 .

Table 1. Potential parameters (atomic units) for the helium rare gas dimers systems

\begin{tabular}{cccccc}
\hline System & $A$ & $b$ & $C_{6}$ & $C_{8}$ & $C_{10}$ \\
\hline $\mathrm{HeHe}$ & 41.96 & 2.523 & 1.461 & 14.11 & 183.6 \\
$\mathrm{HeNe}$ & 98.02 & 2.496 & 3.029 & 36.18 & 545.1 \\
$\mathrm{HeAr}$ & 124.3 & 2.153 & 9.538 & 167.5 & 3701 \\
$\mathrm{HeKr}$ & 118.9 & 2.025 & 13.40 & 280.0 & 7257 \\
$\mathrm{HeXe}$ & 95.90 & 1.853 & 19.54 & 525.0 & 16670 \\
\hline
\end{tabular}

Other potentials for the rare gas dimers are available, in special for the helium diatomic system, which has been studied in more details. For example, Varandas ${ }^{6}$ presented a potential for helium-helium interaction, which has accuracy of one centikelvin at the minimum. Helium diatomic has also been studied by perturbation theory. ${ }^{19}$ Relativistic effects were also incorporated in this sort of molecule. ${ }^{20}$ Although these are very accurate potentials, they are limited to one molecule. The objective of the paper is to study the helium dimers in a comparative phase shift analysis. A critical analysis of available helium dimers potentials deserves a separate study.

The number of bound states the rare gas dimers can support, for different angular momentum, will be presented and discussed. The limit of the phase shift energy for different angular momentum is an integer multiple of $\pi$. Levinson's theorem state this integer is equal to the number of bound states a molecule can support for this angular momentum, ${ }^{3}$

$$
n_{b} \pi=\lim _{E \rightarrow 0} \delta_{l}(E)
$$

From this theorem it is, therefore, possible to use information from the continuum to predict properties about the discrete states.

\section{RESULTS AND DISCUSSION}

Potential for helium molecule, as in Equation 3, predict a energy minimum with $10.99 \mathrm{~K}$ at 5.62 bohrs. In fact the theoretical aspects of the $\mathrm{He}_{2}$ molecule interaction go back to 1928 with a calculation made by Slater. ${ }^{21}$ This 1928 calculation gives dissociation energy and equilibrium distance as $8.9 \mathrm{~K}$ and 5.60 bohrs. Nevertheless, the question of a stable vibrating $\mathrm{He}_{2}$ diatomic molecule was treated in a consistent form only recently.

The existence of helium diatomic molecule is a question of special interest, for it will be the weakest van der Waals molecule. This question was analyzed from a theoretical aspect, using effective range theory, ${ }^{22}$ in which integration of Schrodinger equation is necessary. Since the bound state of such a molecule is reported to be at $10^{-8} \mathrm{eV}$, integration is necessary until $10000 \mathrm{au}$. Therefore, dealing with this energy to detect a bound state can be a source of error. The present method to detect bound states, using the limit of the scattering phase shift, does not have this problem, maximum integration being at $7 \mathrm{au}$.

Convergence of the phase shift for small collision energy has to be investigated to make the Levinson's theorem applicable. This convergence was tested for the HeXe molecule, in which collision energy of about $10^{-10}$ atomic units was obtained. This analysis for a heavy diatomic will guarantee the energy will also be appropriate for the other dimers. Numbers of bound states for the helium dimers are as in Table 2. For angular momentum greater than 5, none of these molecules can support a bound state.

Table 2. Bound state for the helium rare gas dimers systems

\begin{tabular}{ccccccc}
\hline System & $l=0$ & $l=1$ & $l=2$ & $l=3$ & $L=4$ & $l=5$ \\
\hline $\mathrm{HeHe}$ & 0 & 0 & 0 & 0 & 0 & 0 \\
$\mathrm{HeNe}$ & 1 & 1 & 1 & 0 & 0 & 0 \\
$\mathrm{HeAr}$ & 1 & 1 & 1 & 1 & 1 & 0 \\
$\mathrm{HeKr}$ & 2 & 1 & 1 & 1 & 1 & 1 \\
$\mathrm{HeXe}$ & 2 & 1 & 1 & 1 & 1 & 1 \\
\hline
\end{tabular}

Integrating phase shift for low collision energy has to be carried out with a very precise numerical algorithm. The problem arises as a consequence of the Levinson's theorem. In this collision energy limit, phase shift behaves likes a step function, as exemplified for $\mathrm{He}-\mathrm{Kr}$ at zero angular moment and $E=10^{-8} \mathrm{meV}$, in Figure 1. Variable stepsize 
Runge-Kutta of fifth and sixth order as described by Forsythe ${ }^{23}$ were adequate to make relation (4) applicable. For this collision energy a limit for results were to be valid within four significant figures.

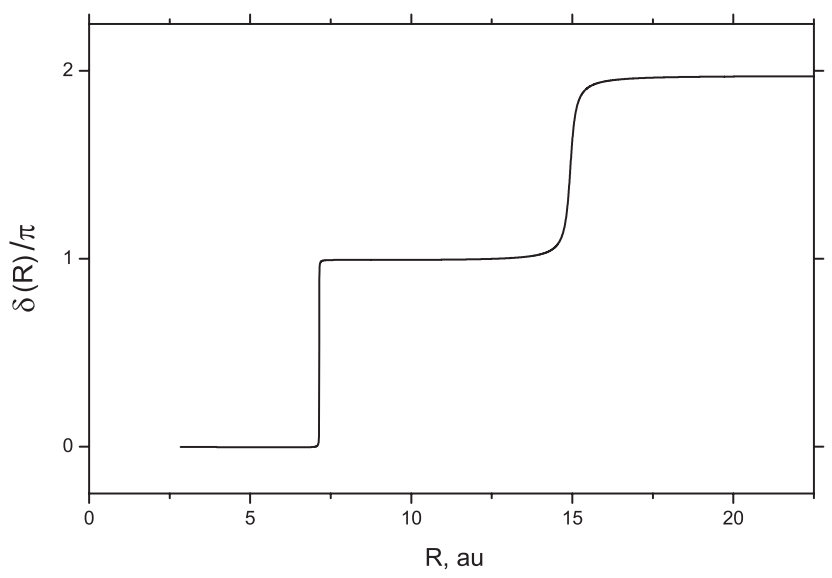

Figure 1. Radial dependence of phase shift for HeKr at zero angular moment and $E=10^{-8} \mathrm{eV}$

A preliminary consideration for the number of bound state can be carried out considering the dissociation energy, which increases by a factor of about $3.0 \times 10^{-5}$ from helium to argon, but increases by $1 / 10$ of this value from Ar to Xe. Different behavior is, therefore, expected from helium to argon and not an appreciable change from argon to xenon, as shown in Table 1. Nevertheless, correlation of dissociation energy with bound states is a crude analysis, for other factor, such as concavity of the potential (force constant) can also play an important role. How the number of bound states will change, for example by a $10 \%$ change in the dispersion coefficient? Sensitivity analysis (SA) provides the answer for this important question.

The sensitivity analysis can be used in two ways: in a direct or inverted procedure. Direct sensitivity analysis was important to discuss the formation of cluster with respect to perturbation in the potential parameter. ${ }^{10}$ Sensitivity analysis was also used to invert and analyze data in quantum scattering, under Born approximation ${ }^{2}$ and in thermodynamic analysis of the second virial data. ${ }^{24-26}$ In the present work SA will be applied to system parameters variation with respect to the formation of the diatomic molecules.

Importance of the sensitivity analysis can be appreciated by considering the radial dependence of phase shift and its derivative, i.e. Equation 2, superimposed by the potential energy itself. These three variables are considered together in Figure 2, for HeXe with $l=0$ and $E=0.1 \mathrm{meV}$.

There is no accumulation of phase in the classical forbidden region. Before $\mathrm{R}=4$ au the potential will have small importance on bound states and collision property. The collision energy to detected bound states is negligible and the turning point is in fact about 6.7 au. This is reflected also in the variable phase equation, showing $\frac{d \delta}{d R}$ has small contribution to be added to the phase.

On the other hand, Equation 2 will go to zero at $\mathrm{E}_{\mathrm{p}}(\mathrm{R})=0$ and will not change sign until convergence. Competition on repulsive and attractive forces will manifest itself in the phase shift. All phase shifts calculated in this work have asymptotic positive values, that is, attractive forces dominate the process to find bound states. The situation is obvious different for larger collision energy.

The region most sensitivity to the potential is around its minimum, in which case the phase derivative has its maximum. Nevertheless, this certainly is not the only region to construct the phase. Phase shift is constructed along the potential, having important contribution from

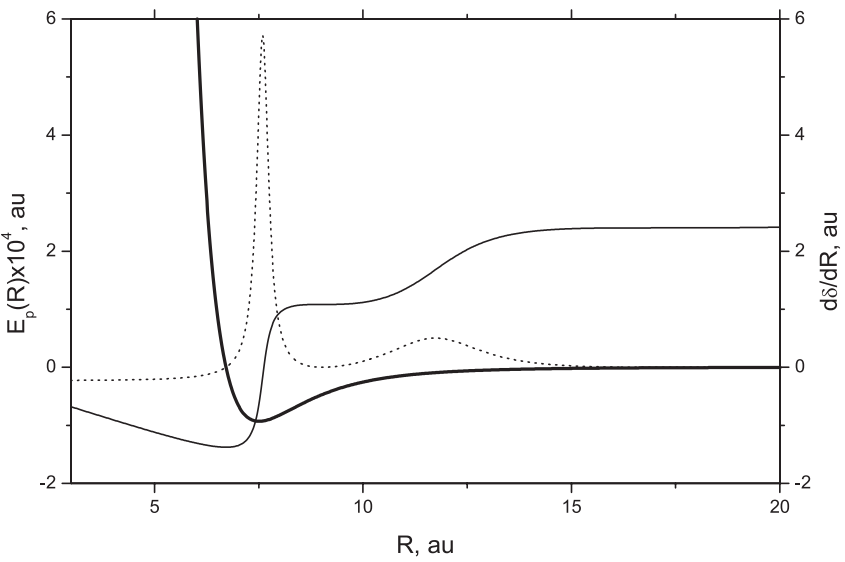

Figure 2. HeXe potential energy function ${ }^{5}$ (thicker continuous line), phase shift (continuous line) and VPM equation (dashed line) for scattering coordinate

the long range part. This preliminary consideration is to be quantified in by varying the potential parameters.

How precise has to be the atomic mass to infer about bound states? Using the helium atomic mass as 4.0026 or $4.002603267 \mathrm{amu}$ will be give zero bound state, indicating the precision of atomic mass is not responsible for the question about $\mathrm{He}_{2}$ stability. Even for the atomic mass equal to $4.02 \mathrm{amu}$ there will be not helium molecule. Similar considerations are also valid for the other members of the series. The number of bound states will not change for HeXe molecule if xenon mass is changed even by $10 \%$. Since atomic masses are precise within one part in ten thousand this parameter can not affect stability of diatomic molecule.

Importance of the dispersion coefficient is to be investigated next. Since long range terms decay as a power of $R^{n}$, one may expect the $C_{10}$ contribution to be less important. The dipole-dipole interaction will have the most important contribution to the molecular spectrum, whereas the quadrupole interaction, $C_{8}$ term, will have a contribution between dipole and octopole. Nevertheless, these coefficients cannot be varied to large values, since this will change the molecule individuality being studied. Small variations around the tabulated values, as in Table 1 , are to be analyzed for zero angular momentum.

For all diatomic molecules in the series, large variations of the $C_{10}$ coefficient do not change the number of bound states with $l=0$. In some cases this coefficient can be set even to zero, except for $\mathrm{HeKr}$. The HeKr has the deepest dissociation energy in the series and this interaction is expected to be more relevant.

On the other hand, a very small variation of the $C_{6}$ coefficient for helium interaction, about $0.46 \%$, will predict a $\mathrm{He}_{2}$ molecule. This is equivalent to change $C_{6}$ from 1.4610 to 1.4678 au. This point represents a $C_{6}$ threshold value for the existence of helium molecule, keeping all other parameters constant. In general, for the three dispersion coefficient and $l=0$, it was found that a maximum variation of $5 \%(\mathrm{HeNe})$ is still accept to give the same number of bound states. This kind of information is important to the inverse problem analysis. Inverted values most are within this range of accuracy.

As the angular momentum is increased dispersion coefficient becomes more important and the repulsive part of the potential less important. Turning points will move to the right in this case. One can qualitatively describe the importance of the short range part of the potential by varying the initial value for integrating the VPM equation. The initial values, without changing the number of bound states are, $3.2 \mathrm{au}(\mathrm{HeNe}), 4.3 \mathrm{au}(\mathrm{HeAr}), 3.1 \mathrm{au}(\mathrm{HeKr})$ and $3.6 \mathrm{au}(\mathrm{HeXe})$. The HeHe molecule has already none bound states and this change in the initial condition will not affect the formation of bound state. Therefore, taking the results for $\mathrm{HeNe}$ as an example, the short range 
potential before $3 \mathrm{au}$ will not affect the bound states. A hard core can be set at this point without change the HeNe spectrum.

\section{CONCLUSIONS}

Quantum scattering sensitivity analysis calculation was carried out for the helium dimers together with bound state structure for different angular momentum. Except for HeHe, all the molecules have bound states. The molecules HeKr and HeXe can support two vibrational states with $l=0$ and these molecules still exist with larger angular momentum. The analysis was performed using the variable phase method and Levinson's theorem.

Sensitivity analysis was carried out to quantify how the phase shift is changed for potentials parameters variations. Mass effect was also carried out for the series. The mass accuracy is not responsible to give a bound state on helium molecule. For example, using atomic mass as 4.0026 or $4.002603267 \mathrm{amu}$ still gives zero bound states for this potential. Changing the mass of xenon atom up to $10 \%$ still leaves $\mathrm{HeXe}$ molecule with two bound states in ground rotational level.

Range of acceptable potential parameters was determined such that the original bound state structure will not be changed. Dispersion coefficients were changed one each time, keeping all other parameters constant. Importance in the dispersion coefficients appears in decreasing order, from $C_{10}$ to $C_{6}$ coefficients. Results are basically unchanged by variation in $C_{10}$ coefficient. On the other hand, variations in $C_{6}$ can be very important. For example, for $\mathrm{He}_{2}$ molecule, a $0.46 \%$ positive variation in this coefficient predicts one vibration state for zero angular momentum. Sensitivity in the repulsive part of the potential was determined by establishing a minimum initial coordinate tolerable to support the original bound state structures. This minimum was found to be around 4.0 au for all the helium dimers.

Although sensitivity analysis was performed in a parametric form for a specific potential, the present results are useful for the bound state rare gas dimers spectrum and for the potential structure. Taking into account the experimental evidence for helium molecule, ${ }^{25}$ one may argue about the potential quality. The present results have shown that, to improve the potential, it is not a matter of changing the potential parameters. Their precision is sufficient to avoid extra spectrum structure. In fact, another functional form of the potential has to be considered, and this can be achieved by the functional sensitivity analysis. ${ }^{26}$ This will provide the correct function for the helium dimers.

\section{ACKNOWLEDGMENT}

We would like to thank CNPq and Fapemig for financial support.

\section{REFERENCES}

1. Aziz, R. A.; J. Chem. Phys. 1993, 99, 4518

2. Lemes, N. H. T.; Borges, E.; Sousa, R. V.; Braga, J. P.; Int. J. Quantum Chem. 2008, 108, 2623.

3. Child, M. S.; Molecular Collision Theory, Academic Press: New York, 1974.

4. Braga, J. P.; Murrell, J. N.; Mol. Phys. 1984, 53, 295.

5. Tang, K. T.; Toennies, J. P.; J. Chem. Phys. 2003, 118, 4976.

6. Varandas, A. J. C.; J. Phys. Chem. A 2010, 114, 8505.

7. Xie, J.; Northby, J. A.; Freeman, D. L.; Doll, J. D.; J. Chem. Phys. 1989, 91,612 .

8. Borges, E.; Ferreira, G. G.; Oliveira, J. M.; Braga, J. P.; Chem. Phys. Lett. 2009, 472, 194.

9. Borges, E.; Ferreira, G. G.; Braga, J. P.; Int. J. Quantum Chem. 2008, 108,2523

10. Berry, R. S.; Chem. Rev. 1993, 93, 2379.

11. Pliego, J. R.; Belchior, J. C.; Braga, J. P.; Phys. Rev. A 1996, 54, 2091.

12. Calogero, F.; Nuovo Cimento 1963, 27, 261.

13. Calogero, F.; Ravenhall, D. G.; Nuovo Cimento 1964, 32, 1755.

14. Calogero, F.; Variable phase approach to potential scattering, Academic Press: New York, 1967

15. Martinazzo, R.; Bodo, E.; Gianturco, F. A.; Comput. Phys. Commun. 2003, 151, 187.

16. Braga, J. P.; Guo, H.; Murrell, J. N.; Dunne, L. J.; Mol. Phys. 1988, 65, 909.

17. Huxley, P.; Knowles, D. B.; Murrell, J. N.; Watts, J. D.; J. Chem. Soc., Faraday Trans. 1984, 80, 1349.

18. Sebastiao, R. C. O.; Lemes, N. H. T.; Virtuoso, L.; Braga, J. P.; Chem. Phys. Lett. 2003, 378, 406.

19. Jeziorska, M.; Cencek, W.; Patkowski, K.; Jeziorski, B.; Szalewicz, K.; J. Chem. Phys. 2007, 127, 124303.

20. Janzen, A.; Aziz, R. A.; J. Chem. Phys. 1997, 107, 914

21. Slater, J. C.; Phys. Rev. 1928, 32, 349.

22. Uang, Y. H.; Stwalley, W. C.; J. Chem. Phys. 1982, 76, 5069.

23. Forsythe, G. E.; Malcolm, M. A.; Moler, C. B.; Computer Methods for Mathematical Computations, Prentice-Hall: Englewood Cliffs, 1977.

24. Lemes, N. H. T.; Sebastiao, R. C. O.; Braga, J. P.; Inv. Prob. Sci. Eng. 2006, 14, 581 .

25. Luo, F.; McBane, G. C.; Kim, G.; Giese, C. F.; Gentry, W. R.; J. Chem. Phys. 1993, 98, 3564.

26. Lemes, N. H. T.; Borges, E.; Braga, J. P.; Chem. Int. Lab. Syst. 2009, 96, 84.

27. Cybulski, S. M.; Toczyłowski, R. R.; J. Chem. Phys. 1999, 111, 10520.

28. Luo F.; MacBane, G. C.; Kilm, G.; Giese, C. F.; Gentry, W. R.; J. Chem. Phys. 1993, 98, 3564 